\title{
Multiple-set split feasibility problems for $\kappa$-asymptotically strictly pseudo-nonspreading mappings in Hilbert spaces
}

\author{
Jing Quan ${ }^{1}$ and Shih-sen Chang ${ }^{2 *}$
}

${ }^{*}$ Correspondence:
changss2013@aliyun.com
${ }^{2}$ College of Statistics and
Mathematics, Yunnan University of
Finance and Economics, Kunming,
Yunnan 650221, China
Full list of author information is
available at the end of the article

${ }^{*}$ Correspondence:

Yunnan 650221, China

available at the end of the article

\begin{abstract}
Some weak and strong convergence theorems for solving multiple-set split feasibility problems for $\boldsymbol{\kappa}$-asymptotically strictly pseudo-nonspreading mappings in infinite-dimensional Hilbert spaces are proved. The results presented in the paper extend and improve the corresponding results of Xu (Inverse Probl. 22(6):2021-2034, 2006), Osilike and Isiogugu (Nonlinear Anal. 74:1814-1822, 2011), Chang et al. (Abstr. Appl. Anal. 2012:491760, 2012), and others.
\end{abstract}

MSC: $47 \mathrm{H05} ; 47 \mathrm{H09}$; 49M05

Keywords: weak and strong convergence; multiple-set split feasibility; $\kappa$-asymptotically strictly pseudo-nonspreading mapping

\section{Introduction}

Throughout this article, we always assume that $H_{1}, H_{2}$ are real Hilbert spaces; ' $\rightarrow$ ' and ' $\rightarrow$ ' denote strong and weak convergence, respectively.

The split feasibility problem $(S F P)$ in finite dimensional spaces was first introduced by Censor and Elfving [1] for modeling inverse problems. The (SFP) can be used in various disciplines such as medical image reconstruction [2], image restoration, computer tomography, and radiation therapy treatment planning [3-5]. The multiple-set split feasibility problem (MSSFP) was studied in [4-7].

Let $A: H_{1} \rightarrow H_{2}$ be a bounded linear operator, $S_{i}: H_{1} \rightarrow H_{1}$ and $T_{i}: H_{2} \rightarrow H_{2}, i=$ $1,2, \ldots, N$, be two finite families of mappings, $C:=\bigcap_{i=1}^{N} F\left(S_{i}\right)$ and $Q:=\bigcap_{i=1}^{N} F\left(T_{i}\right)$, where $F\left(S_{i}\right)$ and $F\left(T_{i}\right)$ are the sets of fixed points of $S_{i}$ and $T_{i}$, respectively.

The so-called multiple set split feasibility problem is

$$
\text { to find } x^{*} \in C \text { such that } A x^{*} \in Q \text {. }
$$

In the sequel, we use $\Gamma$ to denote the set of solutions of the problem (MSSFP) (1.1), that is,

$$
\Gamma=\{x \in C: A x \in Q\}
$$

(02014 Quan and Chang; licensee Springer. This is an Open Access article distributed under the terms of the Creative Commons Attribution License (http://creativecommons.org/licenses/by/2.0), which permits unrestricted use, distribution, and reproduction in any medium, provided the original work is properly cited. 
Let $H$ be a real Hilbert space and $K$ be a nonempty closed convex subset of $H$. Following Kohsaka and Takahashi [8-11], a mapping $T: K \rightarrow K$ is said to be nonspreading if

$$
2\|T x-T y\|^{2} \leq\|T x-y\|^{2}+\|T y-x\|^{2} \quad \text { for all } x, y \in K .
$$

It is to see that the above inequality is equivalent to

$$
\|T x-T y\|^{2} \leq\|x-y\|^{2}+2\langle x-T x, y-T y\rangle \quad \text { for all } x, y \in K \text {. }
$$

In 1967, Browder and Petryshyn [12] introduced the concept of $\kappa$-strictly pseudononspreading mapping.

Definition 1.1 [12] Let $H$ be a real Hilbert space. A mapping $T: D(T) \subset H \rightarrow H$ is said to be $\kappa$-strictly pseudo-nonspreading if there exists $\kappa \in[0,1)$ such that

$$
\|T x-T y\|^{2} \leq\|x-y\|^{2}+\kappa\|x-T x-(y-T y)\|^{2}+2\langle x-T x, y-T y\rangle, \quad \forall x, y \in D(T) .
$$

Clearly, every nonspreading mapping is $\kappa$-strictly pseudo-nonspreading.

The class of asymptotically strict pseudo-contractions was introduced by Qihou [13] in 1996. Kim and Xu [14], Inchan and Nammanee [15], Zhou [16] Cho [17], and Ge [18] proved that the class of asymptotically strict pseudo-contractions is demiclosed at the origin and also obtained some weak convergence theorems for the class of mappings. In 2012, Osilike and Isiogugu [19] introduced a class of nonspreading type mappings which is more general than the class studied in [11] in Hilbert spaces and proved some weak and strong convergence theorems in real Hilbert spaces. Recently, Chang et al. [7] studied the multiple-set split feasibility problem for an asymptotically strict pseudo-contraction in the framework of infinite-dimensional Hilbert spaces.

Definition 1.2 [7] Let $H$ be a real Hilbert space, we say that the mapping $T: D(T) \subset H \rightarrow$ $H$ is a $\kappa$-asymptotically strict pseudo-contraction if there exists a constant $\kappa \in[0,1)$ and a sequence $\left\{k_{n}\right\} \subset[1, \infty)$ with $k_{n} \rightarrow 1(n \rightarrow \infty)$ such that

$$
\left\|T^{n} x-T^{n} y\right\|^{2} \leq k_{n}\|x-y\|^{2}+\kappa\left\|x-T^{n} x-\left(y-T^{n} y\right)\right\|^{2}
$$

holds for all $x, y \in D(T)$.

In this article we introduce the following class of $\kappa$-asymptotically strictly pseudononspreading mappings which is more general than that of $\kappa$-strictly pseudo-nonspreading mappings and $\kappa$-asymptotically strict pseudo-contractions.

Definition 1.3 Let $H$ be a real Hilbert space. A mapping $T: D(T) \subset H \rightarrow H$ is said to be $\kappa$-asymptotically strictly pseudo-nonspreading if there exists a constant $\kappa \in[0,1)$ and a sequence $\left\{k_{n}\right\} \subset[1, \infty)$ with $k_{n} \rightarrow 1(n \rightarrow \infty)$ such that

$$
\begin{aligned}
& \left\|T^{n} x-T^{n} y\right\|^{2} \leq k_{n}\|x-y\|^{2}+\kappa\left\|x-T^{n} x-\left(y-T^{n} y\right)\right\|^{2}+2\left\langle x-T^{n} x, y-T^{n} y\right\rangle, \\
& \forall x, y \in D(T) .
\end{aligned}
$$


Example 1.4 Now, we give an example of $\kappa$-asymptotically strict pseudo-contractive mapping.

Let $C$ be a unit ball in a real Hilbert $l^{2}$, and let $T: C \rightarrow C$ be a mapping defined by

$$
T: \quad\left(x_{1}, x_{2}, \ldots\right) \rightarrow\left(0, x_{1}^{2}, a_{2} x_{2}, a_{3} x_{3}, \ldots\right),
$$

where $\left\{a_{i}\right\}$ is a sequence in $(0,1)$ such that $\prod_{i=2}^{\infty} \alpha_{i}=\frac{1}{2}$.

It is proved in Goebel and Kirk [20] that

(i) $\|T x-T y\| \leq 2\|x-y\|, \forall x, y \in C$;

(ii) $\left\|T^{n} x-T^{n} y\right\| \leq 2 \prod_{i=2}^{n} a_{j}\|x-y\|, \forall n \geq 2$ and $x, y \in C$.

Define $k_{1}^{\frac{1}{2}}=2, k_{n}^{\frac{1}{2}}=2 \prod_{i=2}^{n} a_{j}, n \geq 2$, then

$$
\lim _{n \rightarrow \infty} k_{n}=\lim _{n \rightarrow \infty}\left(2 \prod_{i=2}^{n} a_{j}\right)^{2}=1 .
$$

Letting $\kappa=0$, then $\forall x, y \in C, n \geq 1$, we have

$$
\underline{\left\|T^{n} x-T^{n} y\right\|^{2} \leq k_{n}\|x-y\|^{2}+\kappa\left\|x-y-\left(T^{n} x-T^{n} y\right)\right\|^{2}} .
$$

This implies that $T$ is a $\kappa$-asymptotically strict pseudo-contractive mapping.

Example 1.5 Now, we give an example of $\kappa$-asymptotically strictly pseudo-nonspreading mapping.

Let $X=l^{2}$ with the norm $\|\cdot\|$ be defined by

$$
\|x\|=\sqrt{\sum_{i=1}^{\infty} x_{i}^{2}}, \quad \forall x=\left(x_{1}, x_{2}, \ldots, x_{n}, \ldots\right) \in X,
$$

and let $C=\left\{x=\left(x_{1}, x_{2}, \ldots, x_{n}, \ldots\right) \mid x_{i} \in R^{1}, i=1,2, \ldots\right\}$ be an orthogonal subspace of $X$ (i.e., $\forall x, y \in C$, we have $\langle x, y\rangle=0$ ). It is obvious that $C$ is a nonempty closed convex subset of $X$. For each $x=\left(x_{1}, x_{2}, \ldots, x_{n}, \ldots\right) \in C$, we define a mapping $T: C \rightarrow C$ by

$$
T x= \begin{cases}\left(x_{1}, x_{2}, \ldots, x_{n}, \ldots\right) & \text { if } \prod_{i=1}^{\infty} x_{i}<0 \\ \left(-x_{1},-x_{2}, \ldots,-x_{n}, \ldots\right) & \text { if } \prod_{i=1}^{\infty} x_{i} \geq 0\end{cases}
$$

Next we prove that $T$ is a $\kappa$-asymptotically strictly pseudo-nonspreading mapping.

In fact, for any $x, y \in C$, we have the following cases.

Case 1. If $\prod_{i=1}^{\infty} x_{i}<0$ and $\prod_{i=1}^{\infty} y_{i}<0$, then we have $T^{n} x=x, T^{n} y=y$, and so then inequality (1.3) holds.

Case 2. If $\prod_{i=1}^{\infty} x_{i}<0$ and $\prod_{i=1}^{\infty} y_{i} \geq 0$, then we have that $T^{n} x=x, T^{n} y=(-1)^{n} y$. This implies that

$$
\left\{\begin{array}{l}
\left\|T^{n} x-T^{n} y\right\|^{2}=\left\|x-(-1)^{n} y\right\|^{2}=\|x\|^{2}+\|y\|^{2} \\
k_{n}\|x-y\|^{2}=k_{n}\left(\|x\|^{2}+\|y\|^{2}\right) ; \\
\left\|x-T^{n} x-\left(y-T^{n} y\right)\right\|^{2}=\left[1-(-1)^{n}\right]^{2}\|y\|^{2} ; \\
2\left\langle x-T^{n} x, y-T^{n} y\right\rangle=0 .
\end{array}\right.
$$

Therefore inequality (1.3) holds. 
Case 3. If $\prod_{i=1}^{\infty} x_{i} \geq 0$ and $\prod_{i=1}^{\infty} y_{i} \geq 0$, then we have $T^{n} x=(-1)^{n} x, T^{n} y=(-1)^{n} y$. Hence we have

$$
\left\{\begin{array}{l}
\left\|T^{n} x-T^{n} y\right\|^{2}=\left\|(-1)^{n} x-(-1)^{n} y\right\|^{2}=\|x-y\|^{2}=\|x\|^{2}+\|y\|^{2} ; \\
k_{n}\|x-y\|^{2}=k_{n}\left(\|x\|^{2}+\|y\|^{2}\right) ; \\
\left\|x-T^{n} x-\left(y-T^{n} y\right)\right\|^{2}=\left[1-(-1)^{n}\right]^{2}\|x-y\|^{2}=\left[1-(-1)^{n}\right]^{2}\left(\|x\|^{2}+\|y\|^{2}\right) ; \\
2\left\langle x-T^{n} x, y-T^{n} y\right\rangle=0 .
\end{array}\right.
$$

Thus inequality (1.3) still holds. Therefore the mapping defined by (1.5) is a $\kappa$-asymptotically strictly pseudo-nonspreading mapping.

The purpose of this article is under suitable conditions to prove some weak and strong convergence theorems for solving multiple-set split feasibility problem (1.1) for a $\kappa$-asymptotically strictly pseudo-nonspreading mapping in infinite-dimensional Hilbert spaces. The results presented in the paper extend and improve the corresponding results of Xu [6], Osilike and Isiogugu [19], Chang et al. [7], and many others.

\section{Preliminaries}

In the sequel, we first recall some definitions, notations, and conclusions which will be needed in proving our main results.

Let $E$ be a real Banach space. A mapping $T$ with domain $D(T)$ and range $R(T)$ in $E$ is said to be demiclosed at origin if whenever $\left\{x_{n}\right\}$ is a sequence in $D(T)$ converging weakly to a point $x^{*} \in D(T)$ and $\left\|(I-T) x_{n}\right\|$ converging strongly to 0 , then $T x^{*}=x^{*}$.

A Banach space $E$ is said to have the Opial property if, for any sequence $\left\{x_{n}\right\}$ with $x_{n} \rightarrow x^{*}$, we have

$$
\liminf _{n \rightarrow \infty}\left\|x_{n}-x^{*}\right\|<\liminf _{n \rightarrow \infty}\left\|x_{n}-y\right\|
$$

for all $y \in E$ with $y \neq x^{*}$.

It is well known that each Hilbert space possesses the Opial property.

A mapping $T: K \rightarrow K$ is said to be semicompact if for any bounded sequence $\left\{x_{n}\right\} \subset K$ with $\lim _{n \rightarrow \infty}\left\|x_{n}-T x_{n}\right\|=0$, there exists a subsequence $\left\{x_{n_{i}}\right\} \subset\left\{x_{n}\right\}$ such that $\left\{x_{n_{i}}\right\}$ converges strongly to some point $x^{*} \in K$.

A mapping $T: K \rightarrow K$ is said to be uniformly L-Lipschitzian if there exists a constant $L>0$ such that

$$
\left\|T^{n} x-T^{n} y\right\| \leq L\|x-y\|, \quad \forall x, y \in K .
$$

Let $K$ be a nonempty closed convex subset of a real Hilbert space $H$. The metric projection $P_{K}: H \rightarrow K$ is a mapping such that for each $x \in H, P_{K} x$ is the unique point in $K$ such that $\left\|x-P_{K} x\right\| \leq\|x-y\|, \forall y \in K$. It is known that for each $x \in H$,

$$
\left\langle x-P_{K} x, y-P_{K} x\right\rangle \leq 0, \quad \forall y \in K .
$$

Lemma 2.1 Let $H$ be a real Hilbert space, then the following results hold: 
(i) For all $x, y \in H$ and for all $t \in[0,1]$,

$$
\|t x+(1-t) y\|^{2}=t\|x\|^{2}+(1-t)\|y\|^{2}-t(1-t)\|x-y\|^{2} .
$$

(ii) $\|x+y\|^{2} \leq\|x\|^{2}+2\langle y, x+y\rangle$.

(iii) If $\left\{x_{n}\right\}_{n=1}^{\infty}$ is a sequence in $H$ which converges weakly to $z \in H$, then

$$
\limsup _{n \rightarrow \infty}\left\|x_{n}-y\right\|^{2}=\limsup _{n \rightarrow \infty}\left\|x_{n}-z\right\|^{2}+\|z-y\|^{2}, \quad \forall y \in H .
$$

Lemma 2.2 Let $K$ be a nonempty closed convex subset of a real Hilbert space $H$, and let $T: K \rightarrow K$ be a continuous $\kappa$-asymptotically strictly pseudo-nonspreading mapping. If $F(T) \neq \emptyset$, then it is a closed and convex subset.

Proof Let $\left\{x_{n}\right\} \subset F(T)$ be a sequence such that $\lim _{n \rightarrow \infty} x_{n}=x^{*} \in K$. Now we prove that $x^{*} \in F(T)$. In fact, since $T$ is $\kappa$-asymptotically strictly pseudo-nonspreading, for each $m \geq 1$, we have

$$
\begin{aligned}
\left\|T^{m} x^{*}-x_{n}\right\|^{2}= & \left\|T^{m} x^{*}-T^{m} x_{n}\right\|^{2} \\
\leq & k_{m}\left\|x_{n}-x^{*}\right\|^{2}+2\left(x^{*}-T^{m} x^{*}, x_{n}-T^{m} x_{n}\right\rangle \\
& +\kappa\left\|x^{*}-T^{m} x^{*}-\left(x_{n}-T^{m} x_{n}\right)\right\|^{2} \\
= & k_{m}\left\|x_{n}-x^{*}\right\|^{2}+\kappa\left\|x^{*}-T^{m} x^{*}\right\|^{2} .
\end{aligned}
$$

Taking the limit as $n \rightarrow \infty$ in the above inequality, we have

$$
\left\|T^{m} x^{*}-x^{*}\right\|^{2} \leq \kappa\left\|x^{*}-T^{m} x^{*}\right\|^{2} .
$$

Since $\kappa \in(0,1)$, we have $\left\|T^{m} x^{*}-x^{*}\right\|=0$ for each $m \geq 1$. Hence $T x^{*}=x^{*}$. This shows that $F(T)$ is closed.

Now we prove that $F(T)$ is convex. In fact, let $p_{1}, p_{2} \in F(T)$, and $z=\lambda p_{1}+(1-\lambda) p_{2}$, we prove that $z \in F(T)$. Since $p_{1}-z=(1-\lambda)\left(p_{1}-p_{2}\right)$ and $p_{2}-z=\lambda\left(p_{2}-p_{1}\right)$, by using Lemma 2.1(i), we have

$$
\begin{aligned}
\left\|z-T^{m} z\right\|^{2}= & \left\|\lambda\left(p_{1}-T^{m} z\right)+(1-\lambda)\left(p_{2}-T^{m} z\right)\right\|^{2} \\
= & \lambda\left\|p_{1}-T^{m} z\right\|^{2}+(1-\lambda)\left\|p_{2}-T^{m} z\right\|^{2}-\lambda(1-\lambda)\left\|p_{1}-p_{2}\right\|^{2} \\
\leq & \lambda\left(k_{m}\left\|p_{1}-z\right\|^{2}+\kappa\left\|p_{1}-T^{m} p_{1}-\left(z-T^{m} z\right)\right\|^{2}+2\left\langle p_{1}-T^{m} p_{1}, z-T^{m} z\right)\right) \\
& +(1-\lambda)\left(k_{m}\left\|p_{2}-z\right\|^{2}+\kappa\left\|p_{2}-T^{m} p_{2}-\left(z-T^{m} z\right)\right\|^{2}\right. \\
& \left.+2\left(p_{2}-T^{m} p_{2}, z-T^{m} z\right\rangle\right)-\lambda(1-\lambda)\left\|p_{1}-p_{2}\right\|^{2} \\
= & \lambda\left(k_{m}\left\|p_{1}-z\right\|^{2}+\kappa\left\|z-T^{m} z\right\|^{2}\right)+(1-\lambda)\left(k_{m}\left\|p_{2}-z\right\|^{2}+\kappa\left\|z-T^{m} z\right\|^{2}\right) \\
& -\lambda(1-\lambda)\left\|p_{1}-p_{2}\right\|^{2} .
\end{aligned}
$$

Taking lim $\sup _{m \rightarrow \infty}$ on both sides of the above inequality, we have

$$
\limsup _{m \rightarrow \infty}\left\|z-T^{m} z\right\|^{2} \leq \limsup _{m \rightarrow \infty} \kappa\left\|z-T^{m} z\right\|^{2} .
$$


Since $\kappa<1$, we have

$$
\limsup _{m \rightarrow \infty}\left\|T^{m} z-z\right\|^{2}=0
$$

and so $\lim _{m \rightarrow \infty} T^{m} z=z$, i.e., $T z=z$. This completes the proof.

Lemma 2.3 Let $K$ be a nonempty closed convex subset of a real Hilbert space $H$, and let $T: K \rightarrow K$ be a continuous $\kappa$-asymptotically strictly pseudo-nonspreading mapping. Then $(I-T)$ is demiclosed at 0 , that is, if $x_{n} \rightarrow x^{*}$ and $\lim \sup _{m \rightarrow \infty} \limsup _{n \rightarrow \infty}\left\|\left(I-T^{m}\right) x_{n}\right\|=0$, then $\left\|(I-T) x^{*}\right\|=0$.

Proof Since $\left\{x_{n}\right\}$ is weak convergence, $\left\{x_{n}\right\}$ is bounded. For each $x \in H$, define $f: H \rightarrow$ $[0, \infty)$ by

$$
f(x):=\limsup _{n \rightarrow \infty}\left\|x_{n}-x\right\|^{2}, \quad x \in H
$$

From Lemma 2.1(iii), we have

$$
f(x)=\limsup _{n \rightarrow \infty}\left\|x_{n}-x^{*}\right\|^{2}+\left\|x^{*}-x\right\|^{2}, \quad x \in H .
$$

Thus we have

$$
f(x)=f\left(x^{*}\right)+\left\|x-x^{*}\right\|^{2}, \quad x \in H
$$

In particular, for each $m \geq 1$,

$$
f\left(T^{m} x^{*}\right)=f\left(x^{*}\right)+\left\|T^{m} x^{*}-x^{*}\right\|^{2}
$$

On the other hand, we have

$$
\begin{aligned}
f\left(T^{m} x^{*}\right) & =\limsup _{n \rightarrow \infty}\left\|x_{n}-T^{m} x^{*}\right\|^{2} \\
& =\limsup _{n \rightarrow \infty}\left\|x_{n}-T^{m} x_{n}+T^{m} x_{n}-T^{m} x^{*}\right\|^{2} \\
& =\limsup _{n \rightarrow \infty}\left(\left\|x_{n}-T^{m} x_{n}\right\|^{2}+2\left\langle x_{n}-T^{m} x_{n}, T^{m} x_{n}-T^{m} x^{*}\right\rangle+\left\|T^{m} x_{n}-T^{m} x^{*}\right\|^{2}\right) .
\end{aligned}
$$

Since $\limsup \sup _{m \rightarrow \infty} \limsup _{n \rightarrow \infty}\left\|\left(I-T^{m}\right) x_{n}\right\|=0$ and $T$ is a $\kappa$-asymptotically strictly pseudo-nonspreading mapping, taking $\lim \sup _{m \rightarrow \infty}$ on both sides of the above equality, we get

$$
\begin{aligned}
\limsup _{m \rightarrow \infty} f\left(T^{m} x^{*}\right) \leq & \limsup _{m \rightarrow \infty}\left\|T^{m} x_{n}-T^{m} x^{*}\right\|^{2} \\
\leq & \limsup _{m \rightarrow \infty} \limsup _{n \rightarrow \infty}\left(k_{m}\left\|x_{n}-x^{*}\right\|^{2}+\kappa\left\|x_{n}-T^{m} x_{n}-\left(x^{*}-T^{m} x^{*}\right)\right\|^{2}\right. \\
& \left.+2\left\langle x_{n}-T^{m} x_{n}, x^{*}-T^{m} x^{*}\right\rangle\right) .
\end{aligned}
$$


By virtue of $\limsup _{m \rightarrow \infty} \limsup \sup _{n \rightarrow \infty}\left\|\left(I-T^{m}\right) x_{n}\right\|=0$ and $k_{m} \rightarrow 1(m \rightarrow \infty)$, we have

$$
\limsup _{m \rightarrow \infty} f\left(T^{m} x^{*}\right) \leq f\left(x^{*}\right)+\limsup _{m \rightarrow \infty} \kappa\left\|x^{*}-T^{m} x^{*}\right\|^{2}
$$

On the other hand, it follows from (2.1) that

$$
\limsup _{m \rightarrow \infty} f\left(T^{m} x^{*}\right)=f\left(x^{*}\right)+\limsup _{m \rightarrow \infty}\left\|T^{m} x^{*}-x^{*}\right\|^{2}, \quad \forall x \in H .
$$

Since $\kappa<1$, it follows from (2.2) and (2.3) that $\lim \sup _{m \rightarrow \infty}\left\|T^{m} x^{*}-x^{*}\right\|^{2}=0$. So $\lim _{m \rightarrow \infty} T^{m} x^{*}=x^{*}$ and $T x^{*}=x^{*}$. This completes the proof.

\section{Main results}

Theorem 3.1 Let $H_{1}, H_{2}, A,\left\{S_{i}\right\},\left\{T_{i}\right\}, C, Q$ be the same as in multiple set split feasibility problem (1.1). For each $i=1,2, \ldots, N$, let $T_{i}$ be a uniformly $\tilde{L}_{i}$-Lipschitzian and $\kappa_{i}$ asymptotically strictly pseudo-nonspreading mapping, $S_{i}$ be a uniformly $L_{i}$-Lipschitzian and $\varrho_{i}$-asymptotically strictly pseudo-nonspreading mapping. Let $\left\{x_{n}\right\}$ be the sequence generated by

$$
\left\{\begin{array}{l}
x_{1} \in H_{1} \text { chosen arbitrarily, } \\
u_{n}=x_{n}+\gamma A^{*}\left(T_{n(\bmod N)}^{n}-I\right) A x_{n}, \\
x_{n+1}=\left(1-\alpha_{n}\right) u_{n}+\alpha_{n} S_{n(\bmod N)}^{n} u_{n},
\end{array}\right.
$$

where $\gamma$ is a constant and $\gamma \in\left(0, \frac{1-\kappa}{\lambda}\right), \lambda$ is the spectral of the operator $A^{*} A, \kappa=$ $\max \left\{\kappa_{1}, \kappa_{2}, \ldots, \kappa_{N}\right\}$ and $\left\{\alpha_{n}\right\}$ is a sequence in $(0,1-\varrho]$ with $\varrho=\max \left\{\varrho_{1}, \varrho_{2}, \ldots, \varrho_{N}\right\}$. If $\Gamma \neq \emptyset$, then the sequence $\left\{x_{n}\right\}$ converges weakly to a point $x^{*} \in \Gamma$.

Proof The proof is divided into five steps.

(I) We first prove the limit $\lim _{n \rightarrow \infty}\left\|x_{n}-p\right\|$ exists for any $p \in \Gamma$.

Since $p \in \Gamma$, we have $p \in C:=\bigcap_{i=1}^{N} F\left(S_{i}\right)$ and $A p \in Q:=\bigcap_{i=1}^{N} F\left(T_{i}\right)$. It follows from (3.1) that

$$
\begin{aligned}
\left\|x_{n+1}-p\right\|^{2}= & \left\|u_{n}-p+\alpha_{n}\left(S_{n(\bmod N)}^{n} u_{n}-u_{n}\right)\right\|^{2} \\
= & \left\|u_{n}-p\right\|^{2}+2 \alpha_{n}\left\langle u_{n}-p, S_{n(\bmod N)}^{n} u_{n}-u_{n}\right\rangle \\
& +\alpha_{n}^{2}\left\|u_{n}-S_{n(\bmod N)}^{n} u_{n}\right\|^{2} .
\end{aligned}
$$

Because $S_{i}$ is a $\varrho_{i}$-asymptotically strictly pseudo-nonspreading mapping, for any $v \in H_{1}$, we have

$$
\begin{aligned}
& \left\|S_{n(\bmod N)}^{n} u_{n}-S_{n(\bmod N)}^{n} v\right\|^{2} \\
& \leq \quad\left\|u_{n}-v\right\|^{2}+\varrho\left\|u_{n}-S_{n(\bmod N)}^{n} u_{n}-\left(v-S_{n(\bmod N)}^{n} v\right)\right\|^{2} \\
& \quad+2\left\langle u_{n}-S_{n(\bmod N)}^{n} u_{n}, v-S_{n(\bmod N)}^{n} v\right\rangle .
\end{aligned}
$$

Taking $v=p$, we have

$$
\left\|S_{n(\bmod N)}^{n} u_{n}-p\right\|^{2} \leq\left\|u_{n}-p\right\|^{2}+\varrho\left\|u_{n}-S_{n(\bmod N)}^{n} u_{n}\right\|^{2}
$$


Therefore we have

$$
\begin{aligned}
\left\|S_{n(\bmod N)}^{n} u_{n}-p\right\|^{2} & =\left\|S_{n(\bmod N)}^{n} u_{n}-u_{n}+u_{n}-p\right\|^{2} \\
& =\left\|S_{n(\bmod N)}^{n} u_{n}-u_{n}\right\|^{2}+2\left\langle S_{n(\bmod N)}^{n} u_{n}-u_{n}, u_{n}-p\right\rangle+\left\|u_{n}-p\right\|^{2} \\
& \leq\left\|u_{n}-p\right\|^{2}+\varrho\left\|u_{n}-S_{n(\bmod N)}^{n} u_{n}\right\|^{2} .
\end{aligned}
$$

Simplifying the above inequality, we have that

$$
2 \alpha_{n}\left\langle S_{n(\bmod N)}^{n} u_{n}-u_{n}, u_{n}-p\right\rangle \leq \alpha_{n}(\varrho-1)\left\|u_{n}-S_{n(\bmod N)}^{n} u_{n}\right\|^{2} .
$$

It follows from (3.2) and (3.3) that

$$
\begin{aligned}
& \left\|x_{n+1}-p\right\|^{2} \\
& \quad \leq\left\|u_{n}-p\right\|^{2}+\alpha_{n}(\varrho-1)\left\|u_{n}-S_{n(\bmod N)}^{n} u_{n}\right\|^{2}+\alpha_{n}^{2}\left\|u_{n}-S_{n(\bmod N)}^{n} u_{n}\right\|^{2} \\
& \quad=\left\|u_{n}-p\right\|^{2}-\alpha_{n}\left(1-\varrho-\alpha_{n}\right)\left\|u_{n}-S_{n(\bmod N)}^{n} u_{n}\right\|^{2} .
\end{aligned}
$$

On the other hand,

$$
\begin{aligned}
\left\|u_{n}-p\right\|^{2}= & \left\|x_{n}-p+\gamma A^{*}\left(T_{n(\bmod N)}^{n}-I\right) A x_{n}\right\|^{2} \\
= & \left\|x_{n}-p\right\|^{2}+2 \gamma\left\langle x_{n}-p, A^{*}\left(T_{n(\bmod N)}^{n}-I\right) A x_{n}\right\rangle \\
& +\gamma^{2}\left\|A^{*}\left(T_{n(\bmod N)}^{n}-I\right) A x_{n}\right\|^{2} \\
= & \left\|x_{n}-p\right\|^{2}+2 \gamma\left\langle x_{n}-p, A^{*}\left(T_{n(\bmod N)}^{n}-I\right) A x_{n}\right\rangle \\
& +\gamma^{2}\left\langle A^{*}\left(T_{n(\bmod N)}^{n}-I\right) A x_{n}, A^{*}\left(T_{n(\bmod N)}^{n}-I\right) A x_{n}\right\rangle \\
= & \left\|x_{n}-p\right\|^{2}+2 \gamma\left\langle x_{n}-p, A^{*}\left(T_{n(\bmod N)}^{n}-I\right) A x_{n}\right\rangle \\
& +\gamma^{2}\left\langle A A^{*}\left(T_{n(\bmod N)}^{n}-I\right) A x_{n},\left(T_{n(\bmod N)}^{n}-I\right) A x_{n}\right\rangle \\
\leq & \left\|x_{n}-p\right\|^{2}+2 \gamma\left\langle x_{n}-p, A^{*}\left(T_{n(\bmod N)}^{n}-I\right) A x_{n}\right\rangle \\
& +\gamma^{2}\|A\|^{2}\left\|\left(T_{n(\bmod N)}^{n}-I\right) A x_{n}\right\|^{2} .
\end{aligned}
$$

Since $T_{i}$ is a $\kappa_{i}$-asymptotically strictly pseudo-nonspreading mapping and noting $A p \in$ $\bigcap_{i-1}^{N} F\left(T_{i}\right)$, we have

$$
\begin{aligned}
\left\|T_{n(\bmod N)}^{n} A x_{n}-A p\right\|^{2} & =\left\|T_{n(\bmod N)}^{n} A x_{n}-T_{n(\bmod N)}^{n} A p\right\|^{2} \\
& \leq\left\|A x_{n}-A p\right\|^{2}+\kappa\left\|T_{n(\bmod N)}^{n} A x_{n}-A x_{n}\right\|^{2} .
\end{aligned}
$$

Again since

$$
\begin{aligned}
\left\|T_{n(\bmod N)}^{n} A x_{n}-A p\right\|^{2}= & \left\|T_{n(\bmod N)}^{n} A x_{n}-A x_{n}+A x_{n}-A p\right\|^{2} \\
= & \left\|T_{n(\bmod N)}^{n} A x_{n}-A x_{n}\right\|^{2}+\left\|A x_{n}-A p\right\|^{2} \\
& +2\left\langle T_{n(\bmod N)}^{n} A x_{n}-A x_{n}, A x_{n}-A p\right\rangle,
\end{aligned}
$$


hence from (3.6) and (3.7) we have that

$$
2\left\langle T_{n(\bmod N)}^{n} A x_{n}-A x_{n}, A x_{n}-A p\right\rangle \leq(\kappa-1)\left\|\left(T_{n(\bmod N)}^{n}-I\right) A x_{n}\right\|^{2} .
$$

By virtue of (3.8) we have

$$
\begin{aligned}
& \left\langle T_{n(\bmod N)}^{n} A x_{n}-A x_{n}, T_{n(\bmod N)}^{n} A x_{n}-A p\right\rangle \\
& \quad=\left\langle T_{n(\bmod N)}^{n} A x_{n}-A x_{n}, T_{n(\bmod N)}^{n} A x_{n}-A p+A x_{n}-A x_{n}\right\rangle \\
& =\left\|\left(T_{n(\bmod N)}^{n}-I\right) A x_{n}\right\|^{2}+\left\langle T_{n(\bmod N)}^{n} A x_{n}-A x_{n}, A x_{n}-A p\right\rangle \\
& \quad \leq\left\|\left(T_{n(\bmod N)}^{n}-I\right) A x_{n}\right\|^{2}+\frac{\kappa-1}{2}\left\|\left(T_{n(\bmod N)}^{n}-I\right) A x_{n}\right\|^{2} \\
& =\frac{\kappa+1}{2}\left\|\left(T_{n(\bmod N)}^{n}-I\right) A x_{n}\right\|^{2} .
\end{aligned}
$$

It follows from (3.9) that

$$
\begin{aligned}
2 \gamma & \left\langle x_{n}-p, A^{*}\left(T_{n(\bmod N)}^{n}-I\right) A x_{n}\right\rangle \\
& =2 \gamma\left\langle A\left(x_{n}-p\right),\left(T_{n(\bmod N)}^{n}-I\right) A x_{n}\right\rangle \\
& =2 \gamma\left\langle A\left(x_{n}-p\right)+\left(T_{n(\bmod N)}^{n}-I\right) A x_{n}-\left(T_{n(\bmod N)}^{n}-I\right) A x_{n},\left(T_{n(\bmod N)}^{n}-I\right) A x_{n}\right\rangle \\
& =2 \gamma\left\langle T_{n(\bmod N)}^{n} A x_{n}-A p,\left(T_{n(\bmod N)}^{n}-I\right) A x_{n}\right\rangle-2 \gamma\left\|\left(T_{n(\bmod N)}^{n}-I\right) A x_{n}\right\|^{2} \\
& \leq[\gamma(1+\kappa)-2 \gamma]\left\|\left(T_{n(\bmod N)}^{n}-I\right) A x_{n}\right\|^{2} \\
& =\gamma(\kappa-1)\left\|\left(T_{n(\bmod N)}^{n}-I\right) A x_{n}\right\|^{2} .
\end{aligned}
$$

Substituting (3.10) into (3.5) and then substituting the resulting inequality into (3.4), we have

$$
\begin{aligned}
\left\|x_{n+1}-p\right\|^{2} & \\
\leq & \left\|x_{n}-p\right\|^{2}+\gamma^{2}\|A\|^{2}\left\|\left(T_{n(\bmod N)}^{n}-I\right) A x_{n}\right\|^{2}+[\gamma(\kappa-1)]\left\|\left(T_{n(\bmod N)}^{n}-I\right) A x_{n}\right\|^{2} \\
& \quad-\alpha_{n}\left(1-\kappa-\alpha_{n}\right)\left\|u_{n}-S_{n(\bmod N)}^{n} u_{n}\right\|^{2} \\
\leq & \left\|x_{n}-p\right\|^{2}-\gamma\left(1-\kappa-\gamma\|A\|^{2}\right)\left\|\left(T_{n(\bmod N)}^{n}-I\right) A x_{n}\right\|^{2} \\
& \quad-\alpha_{n}\left(1-\kappa-\alpha_{n}\right)\left\|u_{n}-S_{n(\bmod N)}^{n} u_{n}\right\|^{2} \\
\leq & \left\|x_{n}-p\right\|^{2} .
\end{aligned}
$$

This shows that the $\operatorname{limit}_{n \rightarrow \infty}\left\|x_{n}-p\right\|$ exists.

(II) Now we prove that the $\operatorname{limit}_{n \rightarrow \infty}\left\|u_{n}-p\right\|$ exists.

By (3.11) we have

$$
\begin{gathered}
\gamma\left(1-\kappa-\gamma\|A\|^{2}\right)\left\|\left(T_{n(\bmod N)}^{n}-I\right) A x_{n}\right\|^{2}+\alpha_{n}\left(1-\kappa-\alpha_{n}\right)\left\|u_{n}-S_{n(\bmod N)}^{n} u_{n}\right\|^{2} \\
\leq\left\|x_{n}-p\right\|^{2}-\left\|x_{n+1}-p\right\|^{2} .
\end{gathered}
$$


This implies that

$$
\lim _{n \rightarrow \infty}\left\|\left(T_{n(\bmod N)}^{n}-I\right) A x_{n}\right\|=0,
$$

and

$$
\lim _{n \rightarrow \infty}\left\|u_{n}-S_{n(\bmod N)}^{n} u_{n}\right\|=0
$$

It follows from (3.5), (3.12), and (3.13) that the $\operatorname{limit}_{n \rightarrow \infty}\left\|\lim _{n}-p\right\|$ exists and

$$
\lim _{n \rightarrow \infty}\left\|x_{n}-p\right\|=\lim _{n \rightarrow \infty}\left\|u_{n}-p\right\| .
$$

(III) Now, we prove that $\lim _{n \rightarrow \infty}\left\|x_{n+1}-x_{n}\right\|=0, \lim _{n \rightarrow \infty}\left\|u_{n+1}-u_{n}\right\|=0$.

In fact, it follows from (3.1) that

$$
\begin{aligned}
&\left\|x_{n+1}-x_{n}\right\| \\
&=\left\|\left(1-\alpha_{n}\right) u_{n}+\alpha_{n} S_{n(\bmod N)}^{n} u_{n}-x_{n}\right\| \\
&=\left\|\left(1-\alpha_{n}\right)\left(x_{n}+\gamma A^{*}\left(T_{n(\bmod N)}^{n}-I\right) A x_{n}\right)+\alpha_{n} S_{n(\bmod N)}^{n} u_{n}-x_{n}\right\| \\
&=\left\|\left(1-\alpha_{n}\right)\left(\gamma A^{*}\left(T_{n(\bmod N)}^{n}-I\right) A x_{n}\right)+\alpha_{n}\left(S_{n(\bmod N)}^{n} u_{n}-x_{n}\right)\right\| \\
&=\left\|\left(1-\alpha_{n}\right)\left(\gamma A^{*}\left(T_{n(\bmod N)}^{n}-I\right) A x_{n}\right)+\alpha_{n}\left(S_{n(\bmod N)}^{n} u_{n}-u_{n}\right)+\alpha_{n}\left(u_{n}-x_{n}\right)\right\| \\
&=\|\left(1-\alpha_{n}\right)\left(\gamma A^{*}\left(T_{n(\bmod N)}^{n}-I\right) A x_{n}\right)+\alpha_{n}\left(S_{n(\bmod N)}^{n} u_{n}-u_{n}\right) \\
& \quad+\alpha_{n} \gamma A^{*}\left(T_{n(\bmod N)}^{n}-I\right) A x_{n} \| \\
&=\left\|\gamma A^{*}\left(T_{n(\bmod N)}^{n}-I\right) A x_{n}+\alpha_{n}\left(S_{n(\bmod N)}^{n} u_{n}-u_{n}\right)\right\| .
\end{aligned}
$$

This together with (3.12) and (3.13) shows that

$$
\lim _{n \rightarrow \infty}\left\|x_{n+1}-x_{n}\right\|=0
$$

Similarly, it follows from (3.1), (3.12), and (3.15) that

$$
\begin{aligned}
& \left\|u_{n+1}-u_{n}\right\| \\
& \quad=\left\|x_{n+1}+\gamma A^{*}\left(T_{n+1(\bmod N)}^{n+1}-I\right) A x_{n+1}-\left[x_{n}+\gamma A^{*}\left(T_{n(\bmod N)}^{n}-I\right) A x_{n}\right]\right\| \\
& \quad \leq\left\|x_{n+1}-x_{n}\right\|+\left\|\gamma A^{*}\left(T_{n+1(\bmod N)}^{n+1}-I\right) A x_{n+1}\right\|+\left\|\gamma A^{*}\left(T_{n(\bmod N)}^{n}-I\right) A x_{n}\right\| \\
& \quad \rightarrow 0 \quad(\operatorname{as} n \rightarrow \infty) .
\end{aligned}
$$

(IV) We prove that, for each $j=1,2, \ldots, N$,

$$
\left\|u_{i N+j}-S_{j} u_{i N+j}\right\| \rightarrow 0, \quad\left\|A x_{i N+j}-T_{j} A x_{i N+j}\right\| \rightarrow 0 \quad(i \rightarrow \infty) .
$$

In fact, it follows from (3.13) that

$$
\left\|u_{i N+j}-S_{j}^{i N+j} u_{i N+j}\right\| \rightarrow 0 \quad(i \rightarrow \infty) .
$$


Since $S_{j}$ is uniformly $L_{j}$-Lipschitzian continuous, it follows from (3.16) and (3.18) that

$$
\begin{aligned}
&\left\|u_{i N+j}-S_{j} u_{i N+j}\right\| \\
& \leq\left\|u_{i N+j}-S_{j}^{i N+j} u_{i N+j}\right\|+\left\|S_{j}^{i N+j} u_{i N+j}-S_{j} u_{i N+j}\right\| \\
& \leq\left\|u_{i N+j}-S_{j}^{i N+j} u_{i N+j}\right\|+L_{j}\left\|S_{j}^{i N+j-1} u_{i N+j}-u_{i N+j}\right\| \\
& \leq\left\|u_{i N+j}-S_{j}^{i N+j} u_{i N+j}\right\|+L_{j}\left[\left\|S_{j}^{i N+j-1} u_{i N+j}-S_{j}^{i N+j-1} u_{i N+j-1}\right\|\right. \\
&\left.+\left\|S_{j}^{i N+j-1} u_{i N+j-1}-u_{i N+j}\right\|\right] \\
& \leq\left\|u_{i N+j}-S_{j}^{i N+j} u_{i N+j}\right\|+L_{j}^{2}\left\|u_{i N+j}-u_{i N+j-1}\right\| \\
&+L_{j}\left[\left\|S_{j}^{i N+j-1} u_{i N+j-1}-u_{i N+j-1}\right\|+\left\|u_{i N+j-1}-u_{i N+j}\right\|\right] \\
& \rightarrow 0 \quad(\text { as } n \rightarrow \infty) .
\end{aligned}
$$

Similarly, we can prove that for each $i=1,2, \ldots, N$,

$$
\left\|A x_{i N+j}-T_{j}^{i N+j} A x_{i N+j}\right\| \rightarrow 0 \quad(i \rightarrow \infty) .
$$

Since $T_{j}$ is uniformly $\tilde{L}_{j}$-Lipschitzian continuous, in the same way as above, we can also prove that

$$
\left\|A x_{i N+j}-T_{j} A x_{i N+j}\right\| \rightarrow 0 \quad(\text { as } i \rightarrow \infty) .
$$

(V) Finally, we prove that $x_{n} \rightarrow x^{*}, u_{n} \rightarrow x^{*}$, and it is a solution of problem (MSSFP) (1.1).

In fact, since $\left\{u_{n}\right\}$ is bounded, there exists a subsequence $\left\{u_{n_{i}}\right\} \subset\left\{u_{n}\right\}$ such that $u_{n_{i}} \rightarrow$ $x^{*} \in H_{1}$. Hence, for any positive integer $j=1,2, \ldots, N$, there exists a subsequence $n_{i}(j) \subset n_{i}$ with $n_{i}(j) \bmod N=j$ such that $u_{n_{i}(j)} \rightarrow x^{*}$. Again from (3.17) we have that

$$
\left\|u_{n_{i}(j)}-S_{j} u_{n_{i}(j)}\right\| \rightarrow 0, \quad n_{i(j)} \rightarrow \infty
$$

Since $S_{j}$ is demiclosed at zero, it follows that $x^{*} \in F\left(S_{j}\right)$. By the arbitrariness of $j=1,2, \ldots, N$, we have

$$
x^{*} \in C:=\bigcap_{i=1}^{N} F\left(S_{i}\right) .
$$

Moreover, from (3.1) and (3.13) we have $x_{n_{i}}=u_{n_{i}}-\gamma A^{*}\left(T_{n_{i}(\bmod N)}^{n_{i}}-I\right) A x_{n_{i}} \rightarrow x^{*}$. Since $A$ is a linear bounded operator, it follows that $A x_{n_{i}} \rightarrow A x^{*}$. For any positive integer $k=$ $1,2, \ldots, N$, there exists a subsequence $x_{n_{i}(k)} \subset x_{n_{i}}$ with $n_{i}(k)(\bmod N)=k$ such that $A x_{n_{i}(k)} \rightarrow$ $A x^{*}$ and $\left\|A x_{n_{i}(k)}-T_{k} A x_{n_{i}(k)}\right\| \rightarrow 0$. Since $T_{k}$ is demiclosed at zero, we have $A x^{*} \in F\left(T_{k}\right)$. By the arbitrariness of $k$, it follows that $A x^{*} \in Q:=\bigcap_{k=1}^{N} F\left(T_{k}\right)$. This together with $x^{*} \in C$ shows that $x^{*} \in \Gamma$, that is, $x^{*}$ is a solution to the problem (MSSFP) (1.1).

Next we prove that $x_{n} \rightarrow x^{*}$ and $u_{n} \rightarrow x^{*}$.

In fact, assume that there exists another subsequence $u_{n_{l}} \subset u_{n}$ such that $u_{n_{l}} \rightarrow y^{*} \in \Gamma$ with $y^{*} \neq x^{*}$. Consequently, by virtue of the existence of $\lim _{n \rightarrow \infty}\left\|x_{n}-p\right\|$ and the Opial 
property of a Hilbert space, we have

$$
\begin{aligned}
\liminf _{n_{i} \rightarrow \infty}\left\|u_{n_{i}}-x^{*}\right\| & <\liminf _{n_{i} \rightarrow \infty}\left\|u_{n_{i}}-y^{*}\right\| \\
& =\liminf _{n \rightarrow \infty}\left\|u_{n}-y^{*}\right\| \liminf _{n_{j} \rightarrow \infty}\left\|u_{n_{j}}-y^{*}\right\| \\
& <\liminf _{n_{j} \rightarrow \infty}\left\|u_{n_{j}}-x^{*}\right\|=\liminf _{n \rightarrow \infty}\left\|u_{n}-x^{*}\right\| \\
& =\liminf _{n_{i} \rightarrow \infty}\left\|u_{n_{i}}-x^{*}\right\| .
\end{aligned}
$$

This is a contradiction. Therefore, $u_{n} \rightarrow x^{*}$. By (3.1) and (3.13), we have

$$
x_{n}=u_{n}-\gamma A^{*}\left(T_{n(\bmod N)}^{n}-I\right) A x_{n} \rightarrow x^{*} .
$$

This completes the proof of Theorem 3.1.

Theorem 3.2 Let $H_{1}, H_{2}, A,\left\{S_{i}\right\},\left\{T_{i}\right\}, C, Q$ be the same as in Theorem 3.1. For each $i=1,2, \ldots, N$, let $T_{i}$ be a uniformly $\tilde{L}_{i}$-Lipschitzian and $\kappa_{i}$-asymptotically strictly pseudononspreading mapping, $S_{i}$ be a uniformly $L_{i}$-Lipschitzian and $\varrho_{i}$-asymptotically strictly pseudo-nonspreading mapping. Let $\left\{x_{n}\right\}$ be the sequence generated by

$$
\left\{\begin{array}{l}
x_{1} \in H_{1} \text { chosen arbitrarily, } \\
u_{n}=x_{n}+\gamma A^{*}\left(T_{n(\bmod N)}^{n}-I\right) A x_{n}, \\
x_{n+1}=\left(1-\alpha_{n}\right) u_{n}+\alpha_{n} S_{n(\bmod N)}^{n} u_{n},
\end{array}\right.
$$

where $\gamma$ is a constant and $\gamma \in\left(0, \frac{1-\kappa}{\lambda}\right), \lambda$ is the spectral of the operator $A^{*} A, \kappa=$ $\max \left\{\kappa_{1}, \kappa_{2}, \ldots, \kappa_{N}\right\}$ and $\left\{\alpha_{n}\right\}$ is a sequence in $(0,1-\varrho]$ with $\varrho=\max \left\{\varrho_{1}, \varrho_{2}, \ldots, \varrho_{N}\right\}$. If $\Gamma \neq \emptyset$ and if there exists a positive integer $j$ such that $S_{j}$ is semicompact, then the sequence $\left\{x_{n}\right\}$ converges strongly to a point $x^{*} \in \Gamma$.

Proof Without loss of generality, we can assume that $S_{1}$ is semicompact. It follows from (3.17) that

$$
\left\|u_{n_{i}(1)}-S_{1} u_{n_{i}(1)}\right\| \rightarrow 0, \quad n_{i(1)} \rightarrow \infty
$$

Therefore, there exists a subsequence of $\left\{u_{n_{i}(1)}\right\}$, which (for the sake of convenience) we still denote by $\left\{u_{n_{i}(1)}\right\}$, such that $u_{n_{i}(1)} \rightarrow u^{*} \in H_{1}$. Since $u_{n_{i}(1)} \rightarrow x^{*}, x^{*}=u^{*}$, and so $u_{n_{i}(1)} \rightarrow$ $x^{*} \in \Gamma$. By virtue of $\lim _{n \rightarrow \infty}\left\|x_{n}-p\right\|$ exists, we know that

$$
\lim _{n \rightarrow \infty}\left\|u_{n}-x^{*}\right\|=0, \quad \lim _{n \rightarrow \infty}\left\|x_{n}-x^{*}\right\|=0,
$$

that is, $\left\{u_{n}\right\}$ and $\left\{x_{n}\right\}$ both converge strongly to the point $x^{*} \in \Gamma$. This completes the proof of Theorem 3.2 .

\section{Applications}

In this section we shall utilize the results presented in Section 3 to study the hierarchical variational inequality problem. 
Let $H$ be a real Hilbert space, $S_{i}, i=1,2, \ldots, N$, be uniformly $L_{i}$-Lipschitzian and $\varrho_{i}$ asymptotically strictly pseudo-nonspreading mappings with $\mathcal{F}:=\bigcap_{i=1}^{\infty} F\left(S_{i}\right) \neq \emptyset$. Let $T$ : $H \rightarrow H$ be a nonspreading mapping. The so-called hierarchical variational inequality problem for a finite family of mappings $\left\{S_{i}\right\}$ with respect to the mapping $T$ is to find an $x^{*} \in \mathcal{F}$ such that

$$
\left\langle x^{*}-T x^{*}, x^{*}-x\right\rangle \leq 0, \quad \forall x \in \mathcal{F} .
$$

It is easy to see that (4.1) is equivalent to the following fixed point problem:

$$
\text { find } x^{*} \in \mathcal{F} \text { such that } x^{*}=P_{\mathcal{F}} T x^{*} \text {, }
$$

where $P_{\mathcal{F}}$ is the metric projection from $H$ onto $\mathcal{F}$. Letting $C=\mathcal{F}$ and $Q=F\left(P_{\mathcal{F}} T\right)$ (the fixed point set of $P_{\mathcal{F}} T$ ) and $A=I$ (the identity mapping on $H$ ), problem (4.2) is equivalent to the following multi-set split feasibility problem:

$$
\text { find } x^{*} \in C \text { such that } x^{*} \in Q \text {. }
$$

Hence from Theorem 3.1 we have the following theorem.

Theorem 4.1 Let $H,\left\{S_{i}\right\}, T, C, Q$ be the same as above. Let $\left\{x_{n}\right\},\left\{u_{n}\right\}$ be the sequences defined by

$$
\left\{\begin{array}{l}
x_{1} \in H_{1} \text { chosen arbitrarily, } \\
u_{n}=x_{n}+\gamma(T-I) x_{n}, \quad n \geq 1, \\
x_{n+1}=\left(1-\alpha_{n}\right) u_{n}+\alpha_{n} S_{n(\bmod N)}^{n} u_{n},
\end{array}\right.
$$

where $\gamma$ is a constant and $\gamma \in(0,1)$, and $\left\{\alpha_{n}\right\}$ is a sequence in $(0,1-\varrho]$ with $\varrho=$ $\max \left\{\varrho_{1}, \varrho_{2}, \ldots, \varrho_{N}\right\}$. If $\Gamma \neq \emptyset$, then $\left\{x_{n}\right\}$ converges weakly to a solution of hierarchical variational inequality problem (4.1).

Proof In fact, by the assumption that $T$ is a nonspreading mapping, $T$ is $\kappa$-strictly pseudononspreading with $\kappa=0$. Taking $N=1$ and $A=I$ in Theorem 3.1, by the same method as that given in Theorem 3.1, we can prove that $\left\{x_{n}\right\}$ converges weakly to a point $x^{*} \in \Gamma$, which is a solution of hierarchical variational inequality problem (4.1) immediately.

Competing interests

The authors declare that they have no competing interests.

Authors' contributions

All authors contributed equally and significantly to this research work. All authors read and approved the final manuscript.

Author details

${ }^{1}$ Institute for Mathematics, Yibin University, Yibin, Sichuan 644007, China. ${ }^{2}$ College of Statistics and Mathematics, Yunnan University of Finance and Economics, Kunming, Yunnan 650221, China.

\section{Acknowledgements}

The authors would like to express their thanks to the referees and the editors for their helpful comments and advices. This work was supported by the National Research Foundation of Yibin University (No. 2011B07) and by the Scientific Research Fund Project of Sichuan Provincial Education Department (No. 12ZB345) and the National Natural Sciences Foundation of China (Grant No. 11361170) 


\section{References}

1. Censor, Y, Elfving, T: A multiprojection algorithm using Bregman projections in a product space. Numer. Algorithms 8(2-4), 221-239 (1994)

2. Byrne, C: Iterative oblique projection onto convex sets and the split feasibility problem. Inverse Probl. 18(2), 441-453 (2002)

3. Censor, Y, Bortfeld, T, Martin, B, Trofimov, A: A unified approach for inversion problems in intensity-modulated radiation therapy. Phys. Med. Biol. 51(10), 2353-2365 (2006)

4. Censor, Y, Elfving, T, Kopf, N, Bortfeld, T: The multiple-sets split feasibility problem and its applications for inverse problem and its applications. Inverse Probl. 21(6), 2071-2084 (2005)

5. Censor, Y, Motova, A, Segal, A: Perturbed projections and subgradient projections for the multiple-sets split feasibility problem. J. Math. Anal. Appl. 327(2), 1244-1256 (2007)

6. Xu, HK: A variable Krasnosel'skii-Mann algorithm and the multiple-set split feasibility problem. Inverse Probl. 22(6), 2021-2034 (2006)

7. Chang, S-S, Cho, YJ, Kim, JK, Zhang, WB, Yang, L: Multiple-set split feasibility problems for asymptotically strict pseudocontractions. Abstr. Appl. Anal. 2012, Article ID 491760 (2012). doi:10.1155/2012/491760

8. Kohsaka, F, Takahashi, W: Fixed point theorems for a class of nonlinear mappings relate to maximal monotone operators in Banach spaces. Arch. Math. 91, 166-177 (2008)

9. Kohsaka, F, Takahashi, W: Existence and approximation of fixed points of firmly nonexpansive-type mappings in Banach spaces. SIAM J. Optim. 19, 824-835 (2008)

10. lemoto, S, Takahashi, W: Approximating common fixed points of nonexpansive mappings and nonspreading mappings in a Hilbert space. Nonlinear Anal. 71, 2080-2089 (2009)

11. Kurokawa, Y, Takahashi, W: Weak and strong convergence theorems for nonspreading mappings in Hilbert spaces. Nonlinear Anal. 73, 1562-1568 (2010)

12. Browder, FE, Petryshyn, WV: Construction of fixed points of nonlinear mappings in Hilbert spaces. J. Math. Anal. Appl. 20, 197-228 (1967)

13. Liu, QH: Convergence theorems of the sequence of iterates for asymptotically demicontractive and hemicontractive mappings. Nonlinear Anal., Theory Methods Appl. 26(11), 1835-1842 (1996)

14. Kim, TH, Xu, HK: Convergence of the modified Mann's iteration method for asymptotically strict pseudo-contractions. Nonlinear Anal., Theory Methods Appl. 68, 2828-2836 (2008)

15. Inchan, I, Nammanee, K: Strong convergence theorems by hybrid method for asymptotically image-strict pseudocontractive mapping in Hilbert space. Nonlinear Anal. 3, 380-385 (2009)

16. Zhou, H: Demiclosedness principle with applications for asymptotically pseudo-contractions in Hilbert spaces. Nonlinear Anal. 70, 3140-3145 (2009)

17. Cho, SY: Iterative processes for common fixed points of two different families of mappings with applications. J. Glob. Optim. 57, 1429-1446 (2013)

18. Ge, C: A hybrid algorithm with variable coefficients for asymptotically pseudocontractive mappings in the intermediate sense on unbounded domains. Nonlinear Anal. 75, 2859-2866 (2012)

19. Osilike, MO, Isiogugu, FO: Weak and strong convergence theorems for nonspreading-type mappings in Hilbert spaces. Nonlinear Anal. 74, 1814-1822 (2011)

20. Goebel, K, Kirk, WA: A fixed point theorem for asymptotically nonexpansive mappings. Proc. Am. Math. Soc. 35 $171-174(1972)$

10.1186/1029-242X-2014-69

Cite this article as: Quan and Chang: Multiple-set split feasibility problems for $\kappa$-asymptotically strictly pseudo-nonspreading mappings in Hilbert spaces. Journal of Inequalities and Applications 2014, 2014:69

\section{Submit your manuscript to a SpringerOpen ${ }^{\ominus}$ journal and benefit from:}

- Convenient online submission

- Rigorous peer review

Immediate publication on acceptance

- Open access: articles freely available online

- High visibility within the field

- Retaining the copyright to your article 